\title{
Investigating the correlation of muscle function tests and sarcomere organization in C. elegans
}

Leila Lesanpezeshkia , Hiroshi Qadota ${ }^{b}$, Masoud Norouzi Darabada, Karishma Kashyapc, Carla M. R. Lacerda ${ }^{a}$ Nathaniel J. Szewczyk ${ }^{\text {,e }}$, Guy M. Benian ${ }^{b}$ and Siva A. Vanapallia*

${ }^{a}$ Department of Chemical Engineering, Texas Tech University, Lubbock, TX, 79409, USA

${ }^{b}$ Department of Pathology, Emory University, Atlanta, GA, 30322, USA

${ }^{c}$ Department of Biological Sciences, Texas Tech University, Lubbock, TX, 79409, USA

${ }^{d} \mathrm{MRC} /$ Arthritis Research UK Centre for Musculoskeletal Ageing Research, University of Nottingham, United Kingdom \& National Institute for Health Research Nottingham Biomedical Research Centre, Derby, DE22 3DT, UK

eOhio Musculoskeletal and Neurological Institute (OMNI) and Department of Biomedical Sciences, Ohio University, Athens, $\mathrm{OH}, 45701$, USA

*Corresponding author: Siva. A. Vanapalli, siva.vanapalli@ttu.edu 


\begin{abstract}
Background. Caenorhabditis elegans has been widely used as a model to study muscle structure and function due to many genes having human homologs. Its body wall muscle is functionally and structurally similar to vertebrate skeletal muscle with conserved molecular pathways contributing to sarcomere structure, and muscle function. However, a systematic investigation of the relationship between muscle force and sarcomere organization is lacking. Here, we investigate the contribution of various sarcomere proteins and membrane attachment components to muscle structure and function to introduce $C$. elegans as a model organism to study the genetic basis of muscle strength.
\end{abstract}

Methods. We employ two recently developed assays that involve exertion of muscle forces to investigate the correlation of muscle function to sarcomere organization. We utilized a microfluidic pillar-based platform called NemaFlex that quantifies the maximum exertable force and a burrowing assay that challenges the animals to move in three dimensions under a chemical stimulus. We selected 20 mutants with known defects in various substructures of sarcomeres and compared the physiological function of muscle proteins required for force generation and transmission. We also characterized the degree of sarcomere disorganization using immunostaining approaches.

Results. We find that mutants with genetic defects in thin filaments, thick filaments and M-lines are generally weaker, and our assays are successful in detecting the functional changes in response to each sarcomere location tested. We find that the NemaFlex and burrowing assays are functionally distinct informing on different aspects of muscle physiology. Specifically, the burrowing assay has a larger bandwidth in phenotyping muscle mutants, because it could pick 
ten additional mutants impaired while exerting normal muscle force in NemaFlex. This enabled us to combine their readouts to develop an integrated muscle function score that was found to correlate with the score for muscle structure disorganization.

Conclusions. Our results highlight the suitability of NemaFlex and burrowing assays for evaluating muscle physiology of $C$. elegans. Using these approaches, we discuss the importance of the studied sarcomere proteins for muscle function and structure. The scoring methodology we have developed lays the foundation for investigating the contribution of conserved sarcomere proteins and membrane attachment components to human muscle function and strength.

\section{Keywords}

Muscle physiology / burrowing assay / microfluidics / muscle genetics / sarcomere structure 


\section{Background}

Skeletal muscle, the major contractile tissue in animals, generates the force required for movement. The contractile force generation occurs by the sliding of two filaments composed primarily of the two most abundant muscle proteins - actin and myosin (1). Myosin head domains on the surface of thick filaments exert force by pulling oppositely oriented thin filaments primarily composed of actin inwards from either side, leading to shortening of the fundamental unit of contraction, the sarcomere. Since thousands of sarcomeres are connected end to end to produce myofibrils, and these myofibrils extend the length of the elongated skeletal muscle cell, this force is transmitted longitudinally within a muscle cell ultimately to the tendons and bones, to permit locomotion (1).

The contractile force generation and functional performance of the skeletal muscle can be significantly influenced by genetic defects in the contractile apparatus (2). Mutations such as in ACTN3 and ACE have been shown to improve muscle contractile performance in elite power athletes (3). Alternatively, some disease-causing mutations that impair human muscle function are well known such as in muscular dystrophies $(4,5)$, congenital myopathies $(6)$, and cardiomyopathies (7). Since muscle strength is a useful predictor of all-cause mortality (8), there is a significant interest in uncovering the genetic basis for improved muscle mass (9) and strength (2) with age.

Understanding how genetic mutations influence muscle structure and function is best studied in model organisms (10). Caenorhabditis elegans with 95 body wall muscle cells required for whole animal locomotion is an excellent genetic model to study muscle organization, assembly, 
maintenance, and function $(11,12)$. Its sarcomere is made of dense body and M-line, in which the dense body is analogous to Z-line in humans. Thin filaments are anchored to dense bodies, while thick filaments are organized around M-lines. Actomyosin contractile forces are transmitted to the basement membrane and cuticle through the anchorage of both dense body and $\mathrm{M}$-line that analogously serve the same function of costamere in vertebrate muscle, which are muscle-specific examples of integrin adhesion complexes (13). Unlike vertebrates, the contractile forces in C. elegans are mostly transmitted laterally to the cuticle resulting in bending and sinuous locomotion of the worm on an agar surface (14). In addition, integrin adhesion complexes exist at attachment plaques lying at the boundaries between adjacent muscle cells separated by basement membrane (15), and these also are likely to be involved in force transmission as their absence results in a locomotion defect (16).

The conserved molecular pathways contributing to sarcomere structure, and muscle function have been studied in $C$. elegans using a variety of methods. The animal's transparent body along with immunostaining approaches and various microscopy techniques has helped to visualize the muscle structure $(15,17-21)$. Impairments in muscle function due to genetic defects have been studied by observation of gross phenotypes such as "Unc" (Uncoordinated) adults and "Pat" (Paralyzed arrest at 2-fold) embryonic lethals (13, 17, 22-24). In addition, thrashing and crawling assays have been used to assess muscle function in $C$. elegans $(25,26)$. Despite these advances, how the disorganization in muscle structure, due to genetic defects in the various components of sarcomeres and their membrane attachment structures, influences muscle function remains poorly understood. 
Recently, new methods have been developed in C. elegans that focus on quantitative characterization of muscle function with the capacity to detect more subtle phenotypes in muscle mutants. A bending amplitude assay has been used to identify the locomotion defects in muscle focal adhesion mutants (27). Optogenetic-stimulation has also been used to measure muscle contraction/relaxation rate constants by monitoring body area changes (28). Assessing the animals push and pull forces using atomic force microscopy (29) and pillar deflection measurements in microfluidic devices (30-32) have introduced sophisticated means to calculate the muscle strength of $C$. elegans. Additionally, characterizing the burrowing performance of $C$. elegans where animals move in 3D have enabled assessment of neuromuscular health $(33,34)$.

Studies have begun to employ these new methods to address the relationship between muscle structure and function in C. elegans. Disorganization of actin filaments could support that glucose-treated wild-type animals have reduced thrashing force (32), and electron microscopy of body wall muscle cross-section could justify that the low muscle force is due to disorganization in dense bodies and M-lines (29). However, studies lack a systematic investigation of the relationship between muscle force and structural disorganization which is introduced due to a variety of genetic defects in sarcomere and membrane attachment components. Here, we have utilized two recently developed assays in our laboratory that report on muscle function. The first assay involves measurement of muscle strength using a micropillar-deflection based system called NemaFlex (31) and the second assay involves stimulating animals to burrow in three dimensions in a resistive gel medium (34). We implemented these assays on a set of 20 muscle mutants with genetic defects in various sarcomere components. We also characterized the degree of sarcomere disorganization in 
these mutants and tested the ability of these assays to correlate muscle function to muscle structure. Our results highlight the suitability of our assays and scoring approaches for evaluating $C$. elegans as a genetic model for muscle strength.

\section{Methods}

\section{Worm culture}

Caenorhabditis elegans animals were cultured at $20^{\circ} \mathrm{C}$ on standard nematode growth medium (NGM) on $60 \mathrm{~mm}$ petri plates and never allowed to starve. The NGM plates were seeded with Escherichia coli OP50 bacteria overnight. Age synchronization was done by transferring 20-25 gravid animals to seeded plates and letting them lay eggs for $\sim 3$ hours. The gravid adults were then removed, leaving age synchronized eggs to hatch and develop at $20{ }^{\circ} \mathrm{C}$ until they reach day 1 of adulthood for all the experiments. Day 0 of adulthood is when the age synchronized animals started to lay eggs.

unc-22(sf21), unc-94(sf20), and unc-98(sf19) were generated in and available in the Benian lab. Wild-type, N2(Bristol), and the following mutants were obtained from Caenorhabditis Genetics Center: dyc-1(cx32), pfn-3(tm1362), uig-1(ok884), atn-1(ok84), zyx-1(gk190), unc-95(ok893), tln-1(e259), unc-82(e1220), unc-89(e1460), pkn-1(ok1673), alp-1(tm1137), dim-1(ra102), unc22(e66), unc-54(s95), unc-54(s74), lev-11(x12), and unc-60(r398). Nearly all of these mutants had been multiply outcrossed to wild type. 


\section{Pluronic gel-based burrowing assays}

Burrowing assays were conducted as previously described (34). Briefly, $26 \%$ w/w Pluronic F127 (Sigma-Aldrich) solution was prepared and stored at $4{ }^{\circ} \mathrm{C}$ prior to the experiment to prevent gelation. A minimum number of 30 animals were transferred into the bottom of a Corning $^{\mathrm{TM}}$ Falcon ${ }^{\mathrm{TM}}$ Polystyrene 12 -well plate at $20 \pm 1{ }^{\circ} \mathrm{C}$, either by handpicking them into $20-30$ $\mu \mathrm{L}$ Pluronic solution or in $10 \mu \mathrm{L}$ of worm solution in water, which was then combined with 500 $\mu \mathrm{L}$ of PF-127 to make a base gel layer. Then, the Pluronic layer was cast on top to the thickness of $0.7 \mathrm{~cm}$, followed by $20 \mu \mathrm{L}$ of $100 \mathrm{mg} / \mathrm{mL}$ E. coli solution in liquid $\mathrm{NGM}$ as an attractant ( $\mathrm{t}=0$ min). The number of animals burrowed to the surface was scored every 15 minutes for a total duration of 2 hours. Three replicates per strain were conducted. To compare mutants burrowing performance, two-way ANOVA was used in GraphPad Prism software.

\section{C. elegans muscle strength measurements using NemaFlex}

The muscle strength of $C$. elegans strains was measured using the NemaFlex technique as previously described (31), based on the deflection of soft micropillars as the animals are crawling through the pillar arena. Polydimethylsiloxane (PDMS) devices were poured (Sylgard 184 part $A$ (base) and part B (curing agent) 10:1 by weight; Dow Corning) over the mold by curing for 2.5 hours at $70^{\circ} \mathrm{C}$. The micropillar devices had pillars arranged in a square lattice with a pillar diameter of $44 \mu \mathrm{m}$ and a height of $87 \mu \mathrm{m}$. The gap between the pillars is $71 \mu \mathrm{m}$.

Synchronized day 1 adults were loaded individually in each food-free chamber (35), followed by a 1-minute video collected for each animal at $20 \pm 1{ }^{\circ} \mathrm{C}$. Imaging was performed in brightfield using a Nikon Ti-E microscope with a 4x objective and Andor Zyla sCMOS 5.5 camera at 5 frames 
per second and a pixel resolution of $1.63 \mu \mathrm{m}$ per pixel. Movies were processed and analyzed for strength values using our in-house-built image processing software (MATLAB, R2016a) (Available at https://github.com/VanapalliLabs/NemaFlex). Animal strength was calculated by identifying the pillar with the maximum deflection in each frame to estimate maximal force exerted. We bin these maximal forces and define the animal muscular strength as $f_{95}$, which corresponds to the $95^{\text {th }}$ percentile of these maximal forces. We normalized $f_{95}$ by the cube of animal body diameter to account for differences in animal body diameter (31). The muscle strength of mutants was compared with WT animals by calculating the muscular strength ratio and denoting it as the fold change in muscle strength. Statistical analysis was performed using Wilcoxon rank-sum test in MATLAB.

\section{Immunostaining of body-wall muscle}

Adult nematodes were fixed and immunostained as described previously $(36,37)$. The following primary antibodies were used: anti-ATN-1 (Mouse monoclonal MH35 (19); kindly provided by Dr. Pamela Hoppe, Western Michigan University) and anti-myosin heavy chain A (MHC A; mouse monoclonal 5-6 (38); purchased from the University of lowa Hybridoma Bank) were used at 1:200 dilution, anti-UNC-89 (Rabbit polyclonal EU30) (39)) and anti-UNC-95 (Rabbit polyclonal Benian-13 (40)) were used at 1:100 dilution. Secondary antibodies included antirabbit Alexa 488 (Invitrogen), and anti-mouse Alexa 594 (Invitrogen) both diluted at 1:200. Rhodamine-phalloidin staining of thin filaments was carried out as described (41). Images were captured at room temperature with a Zeiss confocal system (LSM510) equipped with an Axiovert 100M microscope and an Apochromat 63x/1.4 numerical aperture oil immersion 
objective in $2.5 x$ zoom mode. The color balances of the images were adjusted using Adobe Photoshop (Adobe, San Jose, CA).

\section{Muscle disorganization score calculation}

The muscle disorganization score was generated from assessing the immunostaining results of this study (not including others) in which the same methods and antibodies for immunostaining were used. In addition, the scoring was performed independently by two investigators, and the images presented were representative examples from 10 worms for each strain. The discrepancy between two different observers on sarcomere disorganization have been reported to have the coefficient variation of less than $15 \%(42)$.

\section{Results}

\section{Selection of muscle mutants and prior assessment of muscle function}

To investigate the relationship between muscle structure and function in $C$. elegans, we selected 18 muscle proteins (Figure 1). This selection was based on (i) proteins that were located on different structural components of the sarcomere, (ii) mutants that have been previously characterized in terms of locomotion (Table 1), (iii) whether these proteins are involved in generating muscle forces versus transmitting them. With respect to proteins involved in force generation, we selected 2 proteins associated with thick filaments (UNC-22 and UNC-54); and three proteins associated with thin filaments (LEV-11, UNC-60, and UNC-94). With respect to force transmission, we selected 6 proteins localized in dense bodies (UIG-1, 
DYC-1, PFN-3, ATN-1, ALP-1, and DIM-1) and 3 proteins localized in M-lines (UNC-82, UNC-89, UNC-98). We also selected 4 proteins that are localized in both dense bodies and M-lines (ZYX1, TLN-1, UNC-95, PKN-1). Although our selection is limited, targeting various structural units of the sarcomere provides an initial assessment of the role of these proteins in muscle function.

Table 1 shows the list of genes encoding the selected proteins, the particular mutant alleles chosen, indication of the proteins' human homologs, location in the sarcomere, and phenotypic information from locomotory assays reported in the literature. For two genes, unc-54, which encodes the major myosin heavy chain of body wall muscle thick filaments, and unc-60, which

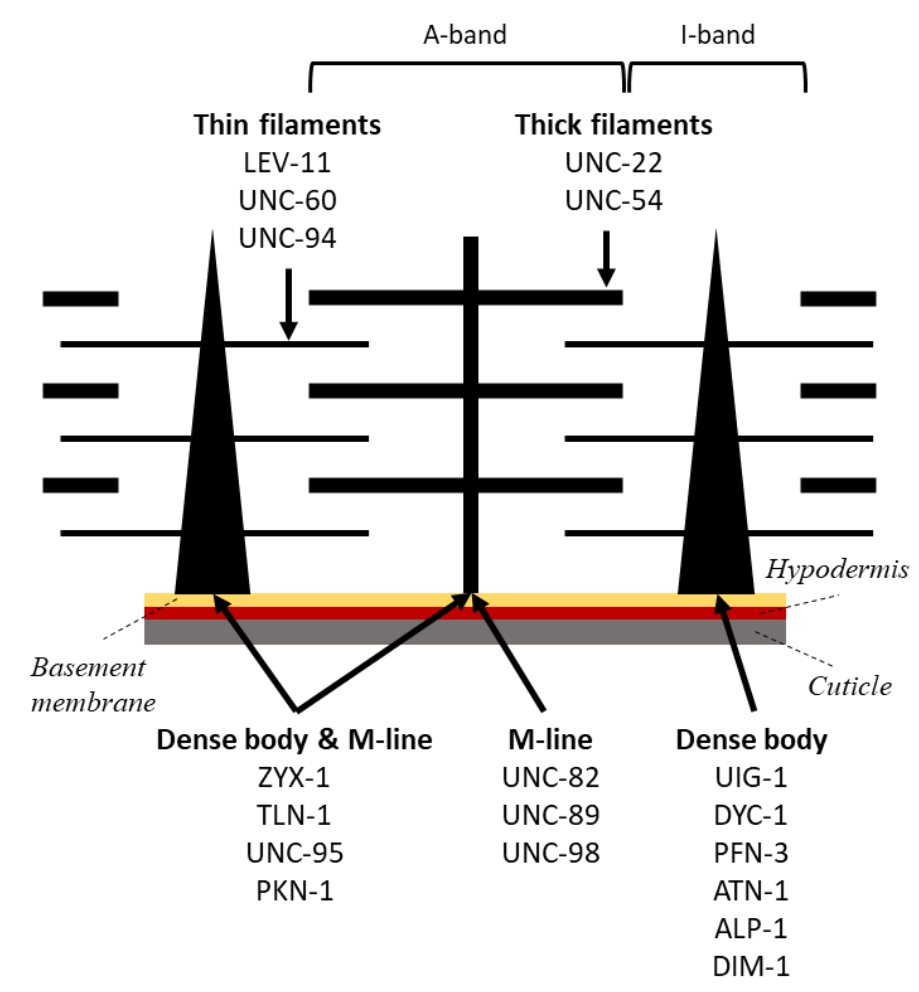

Figure 1: The $C$. elegans sarcomere highlighting the various structural components of the sarcomere. The muscle mutants were selected based on the genes encoding proteins localized to the 5 different structural sites of the muscle: thin filaments, thick filaments, M-line, dense body, and both dense body and M-line. 
encodes ADF/cofilin, we chose to study mild alleles, as the null state for either results in severe paralysis.

The compiled phenotypes include the maximum bending amplitude from a bending assay (27), and the movement rate evaluated from standard swimming and crawling assays. Examining the data, we find that the mutants belonging to M-lines and dense bodies \& M-lines showed slow crawling or swimming movements (Table 1), except for unc-98(sf19) which showed normal crawling but slower movement in swimming. On the contrary, mixed phenotypes were observed for dense bodies, thick filaments, and thin filaments mutants. Some mutants did not show any apparent defects in crawling or swimming assays and some were even better than wild-type (WT) animals. Mutants uig-1, dyc-1, dim-1, and lev-11 were faster in crawling only and unc-22(sf21) was faster in both assays. On the other hand, bending assays identified six of the mutants to be defective in bending (zyx-1, unc-95, pfn-3, atn-1, alp-1, dim-1) and two were normal (unc-98, uig-1). Interestingly, both $t$ In-1 and $d y c-1$ showed exaggerated body bends; however, their movement rates were different with $t / n-1$ crawling slowly, and $d y c-1$ crawling faster. 
Table 1. Summary of the mutants studied, and their human homologs, protein location and reported behavioral phenotypes.

*The crawling assays were conducted under stimulated conditions.

**http://www.wormbase.org, release WS274

\begin{tabular}{|c|c|c|c|c|c|c|}
\hline \multirow{2}{*}{ Mutant } & \multirow{2}{*}{ Type of mutation } & \multirow{2}{*}{ Human homolog } & \multirow{2}{*}{ Protein location } & \multicolumn{3}{|c|}{ Phenotype } \\
\hline & & & & Body bending & Swimming & crawling \\
\hline uig-1(ok884) & deletion, null & PLEKHG1, PLEKHG3 & dense bodies & normal (27) & normal (28) & fast*(28) \\
\hline$p f n-3(t m 1362)$ & deletion, null & profilin & dense bodies & defective (27) & $\begin{array}{c}\text { slightly slow } \\
\text { (43) }\end{array}$ & -- \\
\hline atn-1(ok84) & deletion, null & $\alpha$-actinin & dense bodies & $\begin{array}{c}\text { defective }(27, \\
44)\end{array}$ & slow (28) & normal*(28) \\
\hline alp-1(tm1137) & deletion, null & cypher/ZASP/ oracle & dense bodies & defective (27) & normal (45) & -- \\
\hline$d y c-1(c \times 32)$ & unknown & capon & close to dense bodies & overbent (46) & -- & fast (46) \\
\hline $\operatorname{dim}-1$ (ra102) & $\begin{array}{l}\text { Splice site; loss of } \\
\text { function }\end{array}$ & -- & $\begin{array}{l}\text { around and between } \\
\text { dense bodies }\end{array}$ & defective (27) & slow (28) & fast*(28) \\
\hline$z y x-1(g k 190)$ & deletion, null & Zyxin & dense bodies \& M-lines & defective (27) & -- & -- \\
\hline $\begin{array}{l}\text { tln-1(e259) } \\
\text { previously, } \\
\text { unc-35 }\end{array}$ & unknown & Talin & dense bodies \& M-lines & overbent** & -- & slow (47) \\
\hline unc-95(su33) & nonsense, null & -- & dense bodies \& M-lines & defective (27) & -- & slow (22) \\
\hline$p k n-1$ (ok1673) & deletion, null & protein kinase $\mathrm{N}$ & dense bodies \& M-lines & -- & slow (48) & -- \\
\hline unc-82(e1220) & missense & $\begin{array}{l}\text { ARK5 \& SNARK } \\
\text { protein kinases }\end{array}$ & M-lines & -- & -- & slow (47) \\
\hline unc-89(e1460) & $\begin{array}{l}\text { nonsense in } \\
\text { alternatively spliced } \\
\text { exon; hypomorph }\end{array}$ & obscurin & M-lines & -- & slow (49) & slow (49) \\
\hline unc-98(sf19) & $\begin{array}{c}\text { splice site; } \\
\text { loss of function }\end{array}$ & -- & M-lines & normal (27) & slow $(28,50)$ & normal (28) \\
\hline unc-22(sf21) & missense & titin family member & thick filaments & -- & fast (51) & fast (51) \\
\hline unc-22(e66) & $\begin{array}{l}2 \text { bp deletion; frame- } \\
\text { shift to nonsense; loss } \\
\text { of function }\end{array}$ & titin family member & thick filaments & -- & slow $(28,51)$ & slow (51) \\
\hline unc-54(s95) & missense & myosin heavy chain & thick filaments & -- & -- & slow (52) \\
\hline unc-54(s74) & missense & myosin heavy chain & thick filaments & -- & slow (28) & slow $(28,52)$ \\
\hline lev-11(x12) & $\begin{array}{c}\text { Missense } \\
\text { note: null is Pat } \\
\text { embryonic lethal }\end{array}$ & tropomyosin & thin filaments & -- & normal (28) & fast*(28) \\
\hline unc-60(r398) & $\begin{array}{l}\text { nonsense near C- } \\
\text { terminus; hypomorph }\end{array}$ & ADF/cofilin & thin filaments & -- & slow $(28,53)$ & slow*(28) \\
\hline unc-94(sf20) & nonsense; null & tropomodulin & thin filaments & -- & slow (54) & -- \\
\hline
\end{tabular}




\section{Genetic defects in sarcomeres can reduce $C$. elegans muscle strength and burrowing prowess}

Due to the localization and known function of these proteins in the sarcomere, it is expected that their corresponding loss-of-function mutants would show muscle weakness than WT. However, none of the locomotory assays listed in Table 1 are suitable for measuring muscle strength. Previously, the NemaFlex assay was used to show muscle weakness in unc-17(e245), unc-52(e669), unc-112(r367ts) and multiple alleles of unc-89 $(31,49)$. Here, we have utilized the NemaFlex platform to measure strength of many more muscle mutants listed in Table 1 . Since the body diameter of some of the mutants was significantly different compared to WT (see SI Table S1), we normalized the muscle strength by the cube of the body diameter. This normalization was previously shown to account for the influence of body diameter reasonably well (31) and it also works well for the muscle mutants studied here (see SI Figure S1).

In Figure 2B, we show the normalized strength data for the tested mutants. We find that the mutants with defects in thin filaments, thick filaments and M-lines were generally weaker than WT except lev-11(x12), unc-22(sf21), unc-54(s74) and unc-98(sf19). Animals with genetic impairments in both dense body and M-line did not show a significant difference compared to WT except unc-95(su33). Finally, four out of the six mutants with genetic defects in dense bodies did not show a difference while the other two were weaker than WT. In addition, we find that out of the 20 tested mutants, unc-95 was the weakest. 
A

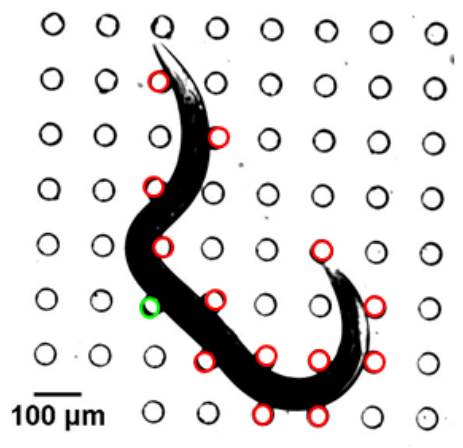

B

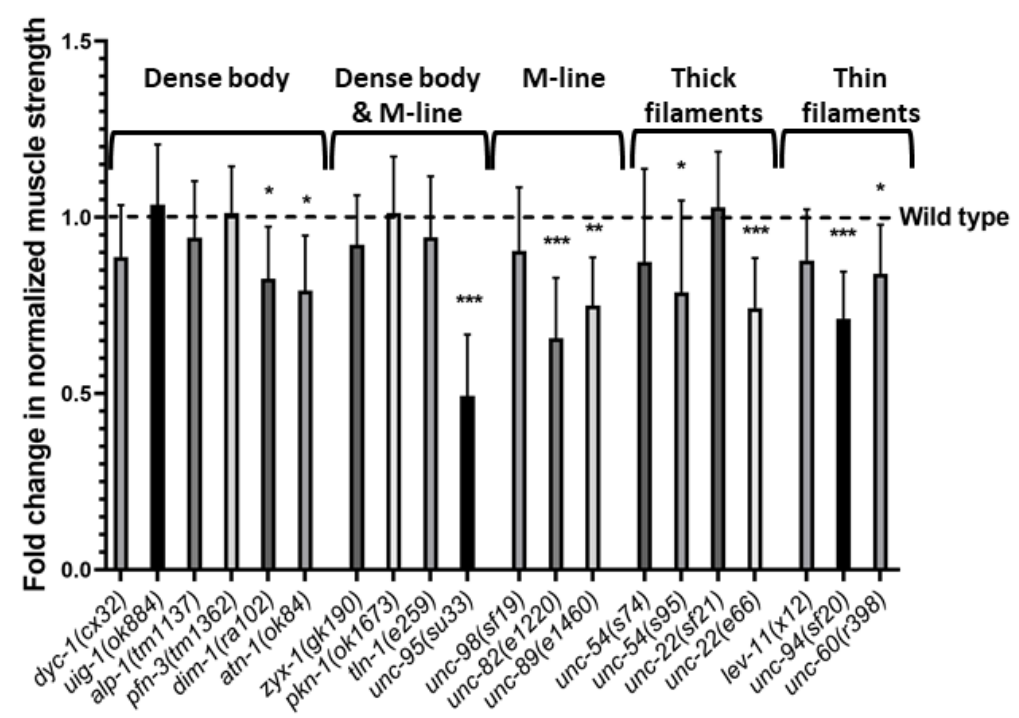

Figure 2: NemaFlex strength measurements on muscle mutants. (A) An animal is crawling in a NemaFlex chamber and deflecting the pillars. The red pillars are in contact with the worm body and undergoing deflection due to muscle forces. The green pillar has the maximal deflection or maximal force exerted by the animal. (B) The fold change in normalized muscle strength of mutants compared to wild-type animals. See Materials and Methods section for estimation of muscle strength from maximal pillar forces and normalization. Error bars represent standard error of the mean. Significance levels are assessed with Wilcoxon rank-sum test, ${ }^{*} P \leq 0.05$, ${ }^{* *} P \leq 0.01$, ${ }^{* * *} P \leq 0.001$. Sample sizes were as following: WT had an average sample size $(\mathrm{N})$ of $30 . \mathrm{N}=36,30,26,25,31,29$ for dyc-1, uig-1, pfn-3, dim-1, atn-1. $\mathrm{N}=20,25,34,20$ for $z y x-1$, pkn-1, t/n-1, unc-95. $\mathrm{N}=27,29,31$ for unc-98, unc-82, unc-89. $\mathrm{N}=36,34,26,31$ for unc-54(s74), unc-54(s95), unc-22(sf21), unc-22(e66). $\mathrm{N}=27,23,27$ for lev-11, unc-94, unc-60.

The results of Figure 2 show that 9 out of 20 tested muscle genes contribute to muscle strength with 6 out of the 9 belonging to genetic defects in M-lines, thick and thin filaments. It appears that genetic defects in these sarcomere components might lead to a more severe loss in muscle strength, thus the assay can detect the physiological consequences of genetic defects in each of these structures. It is interesting to note that the null mutations of 3 components of dense body (uig-1, pfn-3, alp-1) each do not result in severe defects compared to mild mutations of the other structures. Thus it appears that dense body is more resilient to loss of individual components with respect to strength production.

Complementing the NemaFlex assay, we also tested mutants in the burrowing environment where the muscles of $C$. elegans might be challenged differently due to the three-dimensional 
movement $(55,56)$. In the burrowing assay, mutants are loaded at the bottom of well-plate and then stimulated to burrow through the Pluronic gel towards an attractant on the top (Figure $3 A)$. The percentage of the animals that could reach the top are counted at 15-minute intervals for a total duration of 2 hours (34).

The burrowing assay was used previously to show that 4 dense body, and 3 dense body and Mline protein mutants burrow less effectively than WT (34), which led us to evaluate additional muscle mutants with this assay. As shown in Figure 3B-F we find that most of the muscle mutants tested could not burrow as effectively as wild-type, except unc-98(sf19). There was no obvious correlation between the burrowing performance and the structural component where defects were present. Interestingly, none of atn-1(ok84), unc-95(su33) and unc-60(r398) animals could reach the attractant on top, making them amongst the worst burrowers, followed by unc-22(e66) and unc-94(sf20) where only $2 \%$ and $7 \%$ of the animals reached the attractant (Figure 3B).

We have previously shown that this burrowing assay is not merely a chemotaxis assay and in fact, involves exertion of muscles since the burrowing performance varied with gel stiffness and height (34). Also, the mutants that are not defective in 2D chemotaxis (Fig. 6c in reference 34) showed deficiency in burrowing indicating that the muscle actuate differently during the 3D locomotion providing more information on muscle function than 2D chemotaxis assays. This is also confirmed in this study by testing Unc mutants that are known to have uncoordinated movements; however, in a 2D chemotaxis assay, none of unc-22 and unc-54 mutants had any significant impairment to control their movement toward the bacteria in 2D (Figure S2), yet all are defective in burrowing assay. 
Overall, both the NemaFlex and burrowing assays inform on the muscle function of $C$. elegans and highlight that genetic impairments in the muscle can lead to loss of muscle performance.

A

C

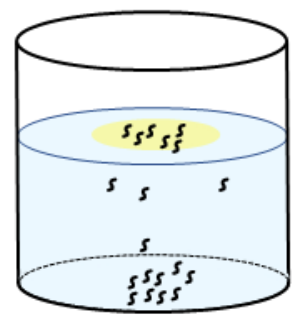

Pluronic gel burrowing assay
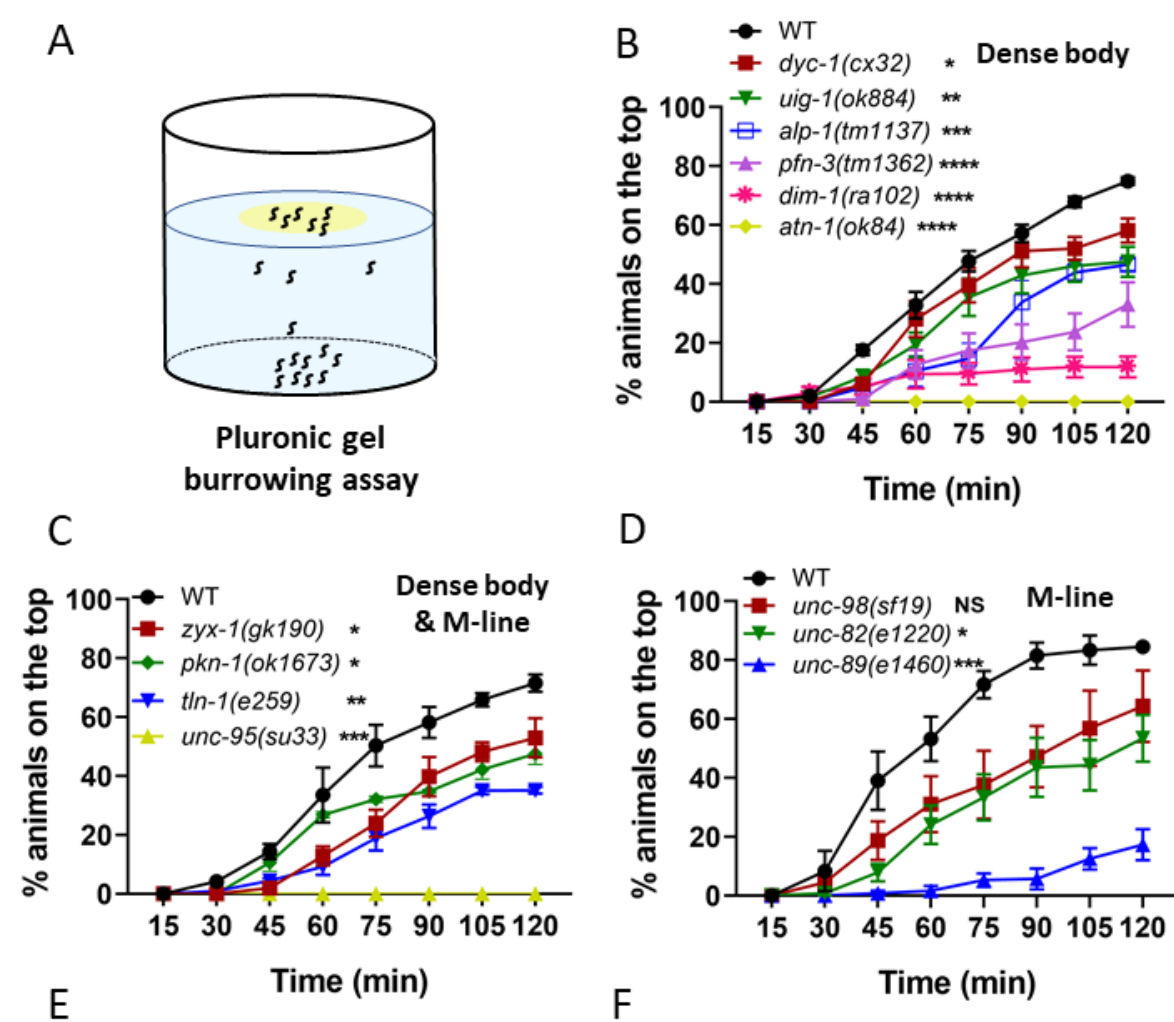

D
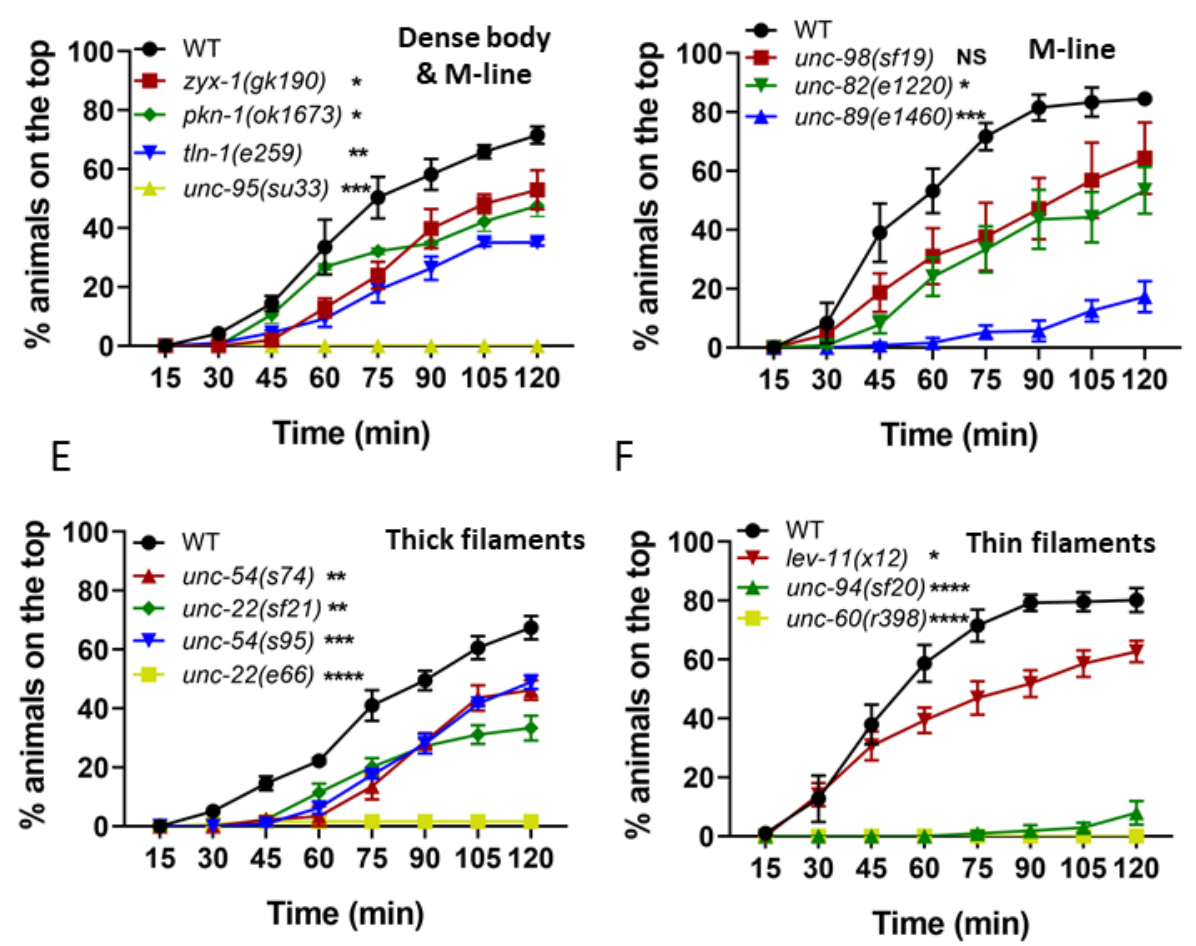

Figure 3: Burrowing performance of muscle mutants. (A) In a Pluronic gel-based burrowing assay, the animals are loaded on the bottom of a well-plate and stimulated to burrow toward an attractant (in yellow) on the top. (B-D) The burrowing performance was assessed by determining the percentage of the animals on the top for 2 hours. The mutants were with genetic defects in the sarcomere genes located on (B) dense body, (C) dense body and M-line, (D) M-line, (E) thick filaments, and (F) thin filaments. Sample sizes were as following: (B) $N=30-44$ for WT, 31-40 for $d y c-1,31-43$ for uig-1, 45-54 for alp-1, 29-39 for pfn-3, 41-48 for dim-1, 29-40 for atn-1. (C) $\mathrm{N}=32-41$ for WT, 30-35 for zyx-1, 35-40 for tIn-1, 33-36 for unc-95. (D) $\mathrm{N}=33-41$ for WT, 26-39 for unc-98, 41-45 for unc-82, 28-41 for unc-89, (E) $\mathrm{N}=45-49$ for WT, 39-56 for unc-54(s74), 44-50 for unc-22(sf21), 41-52 for unc-54(s95), 39-58 for unc-22(e66), (F) $\mathrm{N}=40-48$ for WT, 31-35 for lev-11, 30-35 for unc-94, 34-43 for unc-60. Minimum of 3 replicates were conducted per strain. Error bars are standard error of the mean. Significance levels are assessed with two-way ANOVA. 


\section{Burrowing and NemaFlex strength measures are functionally distinct}

To develop an integral measure of muscle function that combines readouts from NemaFlex and burrowing assay, we first considered whether readouts from these two assays report on distinct aspects of muscle function. The locomotory forces generated by $C$. elegans during twodimensional motion through a mechanical environment consisting of deformable micropillars is expected to be different than those generated during three-dimensional maneuvers made during burrowing, suggesting that these assays might report on unique aspects of muscle function. Indeed, we showed recently that measures extracted from NemaFlex and burrowing for WT animals do not correlate with one another (56). Here, we sought to address the existence or lack of this correlation for the muscle mutants. In addition, we were interested to check if there is data clustering based on the known location of the protein in the sarcomere that is affected by the mutation.

We calculated Z-scores $Z_{j}=\frac{\mu_{j}-\mu_{W T}}{\sigma_{W T}}$ with respect to the wild-type population for each of the functional measures extracted from the NemaFlex and burrowing assay. Here $\mu$ is the mean measured value, which in our case is either the normalized muscle strength (from the NemaFlex assay) or the percent animals reaching the surface at the $2 \mathrm{hr}$ time point (from the burrowing assay), and the subscript $j$ denotes a given mutant strain. Likewise, $\mu w T$ and $\sigma W T$ represent the mean and the standard error of the measured values for WT animals. Thus, a Z-score of 0 indicates that the mutant's muscle function measures were identical to WT.

In Figure 4, we show the Z-scores from the burrowing and NemaFlex assays for all the tested mutants. In the previous section, we showed unc-95, atn-1, unc-60, unc-22(e66), and unc-94 
were the worst burrowers. Consequently, all of these mutants are found on the far left of Figure 4; however, their NemaFlex strength measurements vary from $Z \approx-3$ for unc-60, to the weakest mutant in both NemaFlex and burrowing assays, unc-95, with $Z \approx-8$. On the other hand, dyc-1, uig-1, alp-1, pfn-3, zyx-1, pkn-1, tln-1, unc-98, unc-54(s74), unc-22(sf21), lev-11 that were not significantly different than WT in strength measurement lie toward the top right side of Figure 4 with a large range of burrowing Z-score between -19 for unc-22(sf21) to -4.8 for unc-98. This suggests that the muscle function impairments are more pronounced in burrowing assay. Overall, this data shows that these two assays are modestly correlated (Spearman correlation coefficient is 0.42 ) which is expected since both involve actuation of muscles, but given that they are not completely correlated also suggests that they can inform on different aspects of muscle physiology. We also could not find any evidence of clustering based on the locations of the proteins affected.

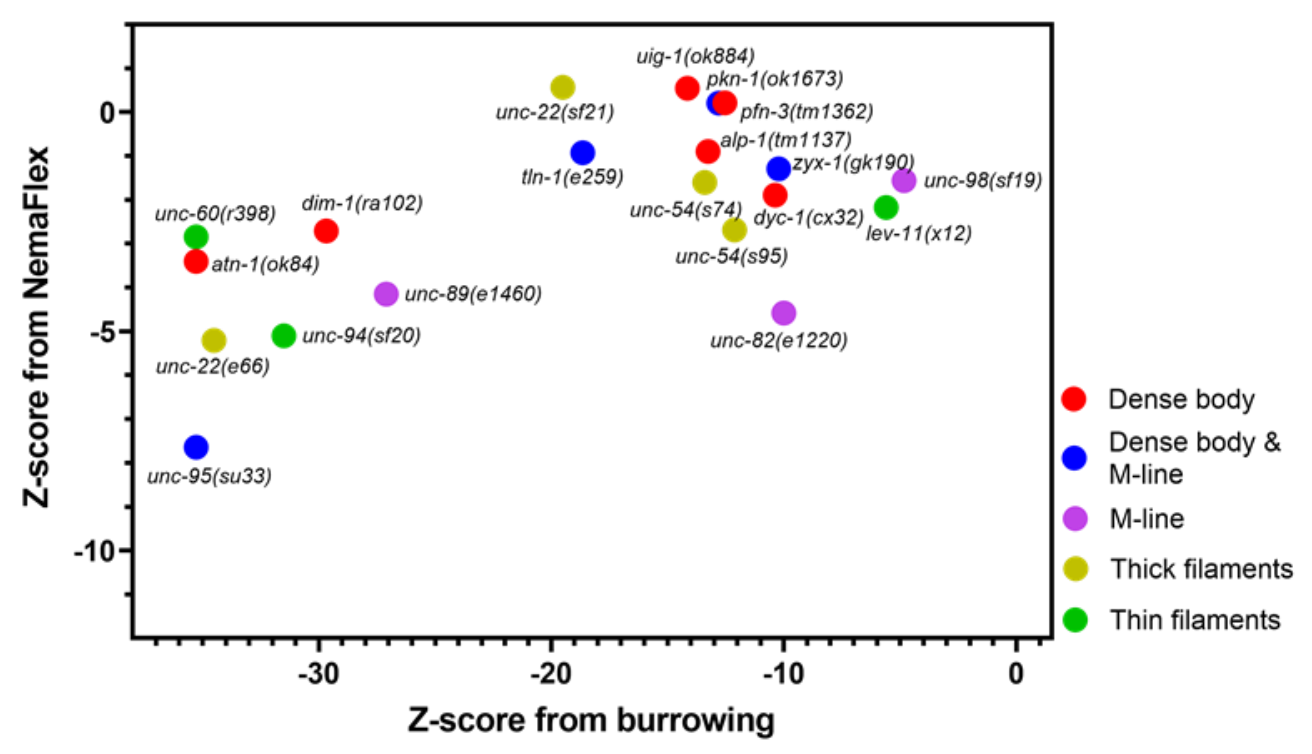

Figure 4: Burrowing assay and NemaFlex strength measurement are not correlated strongly. Z-score of 0 represents being identical to WT. The mutants are color coded based on the protein location in the sarcomere. 


\section{A scoring system for structural disorganization of $C$. elegans muscle}

Structural disorganization in the sarcomeres can adversely affect the contraction-relaxation cycle consequently interfering with muscle function. As NemaFlex and burrowing assays engage C. elegans in actuating its muscles, we speculated that the mutants with a low Z-score in at least one of the assays are likely to exhibit some level of disorganization in the sarcomere structure. We therefore performed immunostaining to visualize the structural disorganization in the muscle contractile apparatus of mutants and developed a scoring system to rank the level of disorganization.

Dense body, M-line, A-band, and I-band are the main substructures of the sarcomere, the fundamental unit of muscle contraction. Thus, we evaluated sarcomeric organization at these sites by immunostaining with antibodies to the following proteins: MHCA (myosin heavy chain A) that is located in the middle of A-bands which represent assembled and organized myosin thick filaments, UNC-95 that is located at the bases of dense bodies and M-lines, UNC-89 that is located throughout the depth of the M-lines, and ATN-1 that is located in the major but not basal portion of dense bodies. Phalloidin was also used to stain I-bands where actin thin filaments are located. We also surveyed the literature and collected images of the aforementioned muscle structures of the same allele used in this study. Table 2 shows the muscle structure data for 20 mutants with 69 data points obtained from this study, and 36 collected from literature. The immunostained images for the mutants obtained in this study are shown in SI Figure S3. 
Table 2. Muscle Disorganization Score (MDS). Each of the structural sites were given a score from 0 to 3 , based on the severity of disorganization. MDS for a mutant is the sum of these scores on each site. Left panel refers to WT with normal muscle structure and MDS $=0$, while this score is 14 for unc-60(r398) due to the severity of its muscle disorganization.

${ }^{*}$ Immunostaining results reported in (Hoppe et al. 2010) were obtained on a different allele, unc82(e1323), which is a premature stop codon and is likely a null allele.

\begin{tabular}{|cc|}
\hline Muscle disorganization score \\
\hline Normal & $\mathbf{0}$ \\
\hline Mild & 1 \\
\hline Moderate & $\mathbf{2}$ \\
\hline Severe & 3 \\
\hline
\end{tabular}

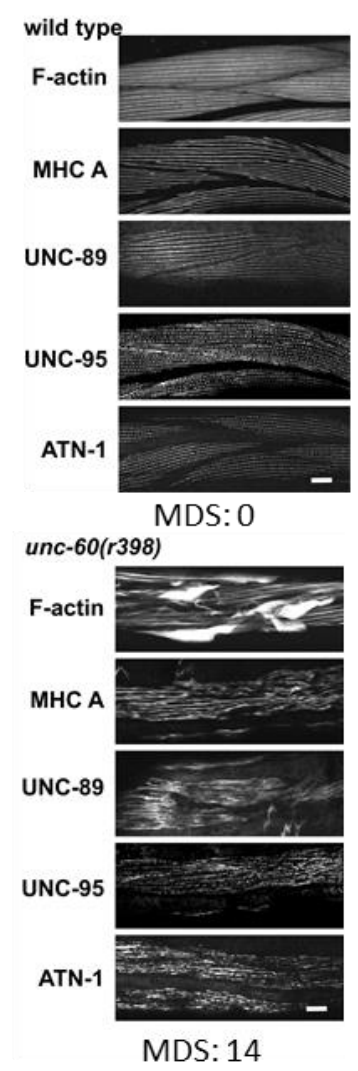

\begin{tabular}{|c|c|c|c|c|c|c|}
\hline & $\begin{array}{l}\text { Phalloidin } \\
\text { (I-band) }\end{array}$ & $\begin{array}{c}\text { MHCA } \\
\text { (A-band) }\end{array}$ & $\begin{array}{l}\text { UNC-95 (base } \\
\text { of dense body } \\
\text { \& M-line) }\end{array}$ & $\begin{array}{l}\text { UNC-89 } \\
\text { (M-line) }\end{array}$ & $\begin{array}{c}\text { ATN-1 } \\
\text { (Dense } \\
\text { body) }\end{array}$ & MDS \\
\hline Wild type & 0 & 0 & 0 & 0 & 0 & 0 \\
\hline uig-1(ok884) & 1 & 1 & 1 & 1 & 1 & 5 \\
\hline$p f n-3(t m 1362)$ & 0 & 1.5 & 0 & 0 & 1 & 2.5 \\
\hline atn-1(ok84) & $2(44)$ & 0 & $2(15)$ & $0(15)$ & $3(44)$ & 7 \\
\hline alp-1(tm1137) & 1, in $9 \%(45)$ & $0(45)$ & 0 & 0 & $0(45)$ & 1 \\
\hline$d y c-1(c \times 32)$ & 0 & 0 & 0 & 1 & 0 & 1 \\
\hline $\operatorname{dim}-1$ (ra102) & 2 & 3 & 1 & 2 & 1 & 9 \\
\hline$z y x-1(g k 190)$ & 0 & 0 & 0 & 0 & 0 & 0 \\
\hline $\operatorname{tln}-1(e 259)$ & 0 & 1 & 0 & 0 & 0 & 1 \\
\hline unc-95(su33) & $3(57)$ & $3(57)$ & 3 (PAT-3) (57) & 3 & 3 & 15 \\
\hline$p k n-1$ (ok1673) & $0(48)$ & $0(48)$ & $0(48)$ & 0 & $0(48)$ & 0 \\
\hline unc-82(e1220) & $2 *(58)$ & $3(58)$ & 2 & $3(58)$ & $0(58)$ & 10 \\
\hline unc-89(e1460) & 2 & 3 (unpub) & 2 & $3(39)$ & 1 & 11 \\
\hline unc-98(sf19) & 1 & $2(59)$ & $0(15)$ & $2(50)$ & 1 & 6 \\
\hline unc-22(sf21) & $0(51)$ & $0(51)$ & $0(51)$ & 0 (unpub) & 0 (unpub) & 0 \\
\hline unc-22(e66) & 2 & $2(21)$ & $2(21)$ & $2(21)$ & $2(21)$ & 10 \\
\hline unc-54(s95) & 0 & 1 & 1 & 1 & 1 & 4 \\
\hline unc-54(s74) & 0 & 1 & 2 & 1 & 1 & 5 \\
\hline lev-11(x12) & 0 & 1 & 0 & 0 & 0 & 1 \\
\hline unc-60(r398) & 3 & 3 & 2 & 3 & 3 & 14 \\
\hline unc-94(sf20) & $2(54)$ & $1(54)$ & 2 & $1(54)$ & $1(54)$ & 7 \\
\hline
\end{tabular}

The mutants were evaluated based on the level of structural disorganization of each of the stained structures and were assigned a score from 0 to 3 for normal to the most severe disorganization (Table 2). Muscle Disorganization Score (MDS) is defined as the sum of the disorganization score of each individual structure. A mutant can acquire a value ranging from 0 
for an organized structure to a maximum of 15 for severely disorganized muscle structure. The left panel of Table 2 illustrates two examples on how MDS is calculated: WT animals or the control group received an MDS $=0$, as none of the structures studied were disorganized, and unc-60(r398) was given MDS = 14 because of the severe structural disorganization observed on I-bands, A-bands, and the depth of M-lines and dense bodies, along with the moderate disorganization on the base of dense bodies and M-lines.

With respect to mutants, we did not observe any disorganization in zyx-1, pkn-1, unc-22(sf21) body wall muscle structure (Table 2). Interestingly, none of these mutants were weaker in NemaFlex strength measurements, yet they were all burrowing impaired. Likewise, the mutants with low Z-scores on both NemaFlex and burrowing shown on the bottom left corner of Figure 4, had structurally disorganized muscles; i.e. MDS of unc-95 and unc-22(e66) were 15 and 10, respectively. In the next section, we use this scoring system to understand how muscle structure and muscle function are related to each other.

\section{Correlating muscle function with muscle structure disorganization}

To understand how structural organization of the myofilament lattice can contribute to muscle function, we sought a functional readout characterizing muscle physiology. Since NemaFlex and burrowing Z-score values were not in the same range, we normalized them with respect to the absolute maximum so that the normalized Z-scores $\bar{Z}_{j}$ lie in the range $-1<\bar{Z}_{j}<0$. In Figure $5 \mathrm{~A}$ and Figure 5B, we plot the normalized NemaFlex and burrowing scores as a function of the muscle disorganization score. In general, both the normalized NemaFlex and burrowing scores decrease with increase in the score of muscle structure disorganization. Principal component 
analysis (PCA) suggested that the summation of the normalized NemaFlex and burrowing Zscores with almost the same weight, can result in a new latent variable, which we refer to as muscle function score that could explain $80 \%$ of data variation (See SI Note S1). Figure 5 C shows that the correlation using this new muscle function is improved compared to each individual physiological Z-score. Interestingly, all data points fall inside the $95 \%$ prediction bound of the fitted line. Overall, these results suggest that the lower the muscle function score is, the greater is the degree of sarcomeric disorganization, indicating these two muscle physiology assays together can improve the chance of successfully detecting any structural disorganizations in the animal's body wall muscle.

Additionally, Figure $5 \mathrm{C}$ shows that all the $\mathrm{M}$-line and thick filaments mutants are mildly disorganized in their muscle structure with mixed physiological functions. The muscle structure of dense body mutants varies from nearly normal values to mild disorganization and also normal to mild physiological function. Most of the dense body \& M-line mutants have almost well-organized muscle structures with normal physiological performance, except for unc-95 that was both structurally and physiologically severely impaired. For mutants with defects in thin filaments, muscle structural disorganization and muscle function vary from mild to severe. 

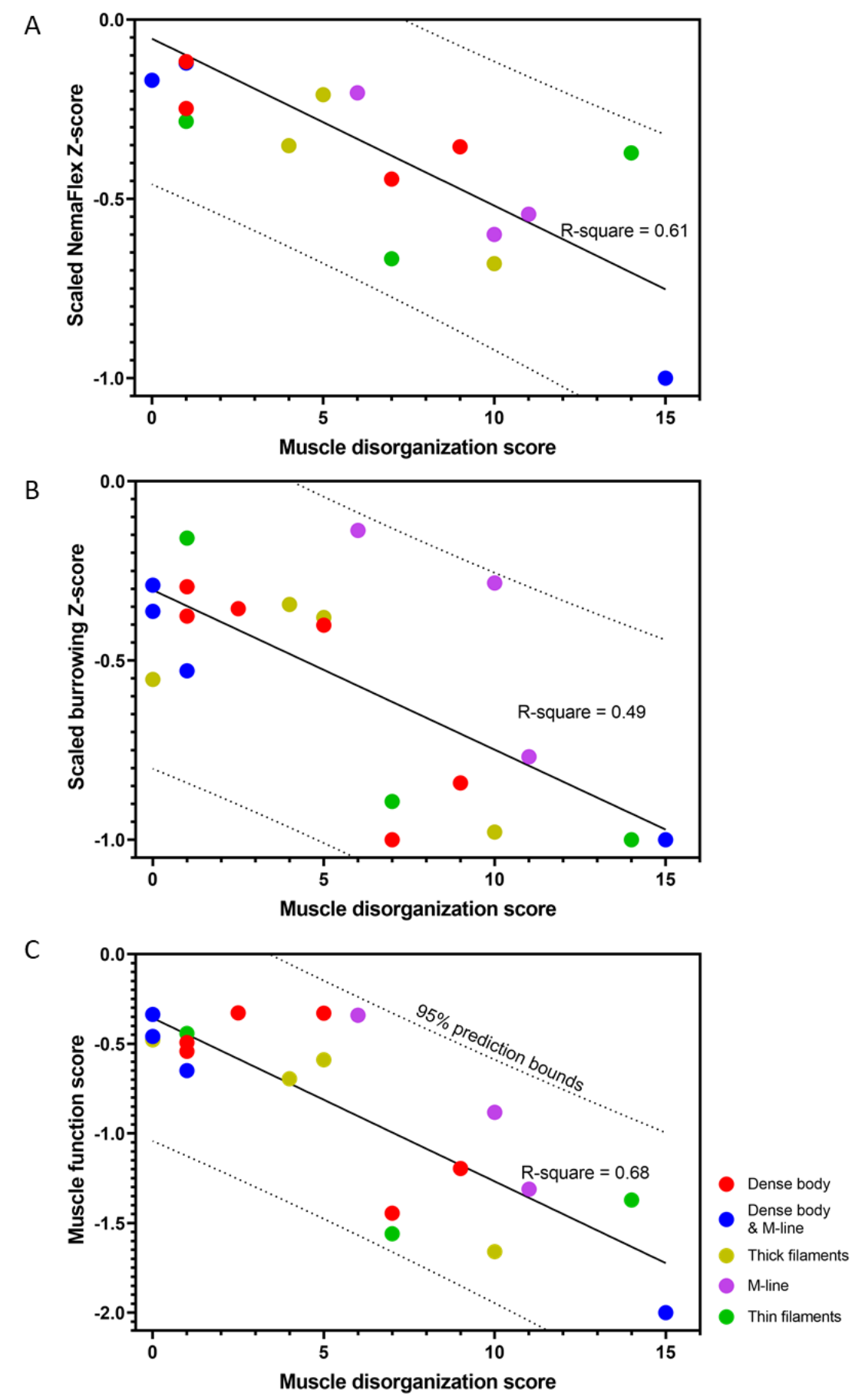

Figure 5: Muscle function score is correlated with muscle disorganization score. (A,B) NemaFlex and Burrowing Z-score values show the $R$-square of 0.61 and 0.49 respectively from linear regression. (C) Muscle function score is the summation of scaled Z-scores from NemaFlex and burrowing assays. The black line shows the linear regression fit with R-square of 0.68 and the $95 \%$ prediction bounds are shown as dotted lines. 


\section{Discussion}

\section{Novel assays advance studies of muscle function in C. elegans}

Our results demonstrate the effectiveness of two novel assays - NemaFlex and burrowing - in detecting functional deficiencies in C. elegans muscle mutants. Furthermore, our results suggest that each of the assays informs on different aspects of muscle physiology. Thus, assays in which C. elegans response is measured in 2D and 3D environments can provide unique information on muscle function.

Both the NemaFlex and burrowing assays showed 9 mutants out of 20 being defective. This includes dim-1 and atn-1 with defects in dense body, unc-95 with a defect in dense body and M-line, unc-82 and unc-89 with defects in M-line, unc-54(s95) and unc-22(e66) with defects in thick filaments, and unc-94 and unc-60 with defects in thin filaments (Figures 2 and 3). Apart from these mutants, there were 10 other mutants that were only burrowing impaired. This shows that the burrowing assay has a larger bandwidth in phenotyping muscle mutants with subtle genetic defects which are not detected by NemaFlex.

Comparing the outcomes from standard locomotory assays of swimming or crawling, we observe that out of the 20 muscle mutants, except four mutants uig-1, dyc-1, unc-22(sf21) and lev-11(x12), the rest are defective in either crawling or swimming (Table 1). 9 out of the 20 muscle mutants that were found to be defective in both NemaFlex and burrowing were also found to be defective in swimming or crawling. Interestingly, among the four mutants showing a fast phenotype in either the crawling or swimming assay, uig-1, dyc-1, unc-22(sf21) and lev- 
11(x12) were significantly burrowing impaired, yet none of them were weaker in NemaFlex muscle strength assessment.

Overall, comparing the results from NemaFlex and burrowing assays to the traditional swimming and crawling assays suggests that the common impairments among all these assays are mostly associated with the mutations of the related proteins found on M-line in 2 mutants, dense body and $\mathrm{M}$-line in 1 mutant, thick filaments and thin filaments each in 2 mutants. Mixed phenotypes were identified in dense body mutants, as there was no concurrence in atn-1 and dim-1 swimming and crawling assays, yet both were impaired in NemaFlex and burrowing.

\section{Correlating muscle function to muscle structure}

We investigated mutants with genetic defects in their muscle structure with the aim of relating structural disorganization in sarcomeres to muscle function. We built a scoring system to evaluate the muscle structural disorganization by immunostaining key sarcomeric structural components including dense body, M-line, A-band, and I-band. We also developed a muscle function score by combining the outputs of both NemaFlex and burrowing assays to result in a score between -2 for the most impaired animals and 0 for the animals that were physiologically similar to WT.

Our results indicate that the muscle function score is negatively correlated with muscle disorganization score (Figure 5C). Unc-95, unc-60, unc-89, unc-82, and unc-22(e66) were the mutants with the highest level of disorganized body wall muscles with muscle disorganization score of $15,14,11,10,10$, respectively. These mutants can be found on the lower right of Figure 5C, correlated with low muscle function score justifying their impaired muscle function. 
Interestingly, atn-1 and unc-94 were found to be physiologically impaired (score of $\sim-1.5$ out of the minimum -2), yet their muscle structure was only mildly disorganized.

Mutants zyx-1, pkn-1, unc-22(sf21) were found to have a muscle disorganization score of 0 , and dyc-1, alp-1, t/n-1, and lev-11 had a score of 1 that were well correlated with the high scores of muscle function. The reason why these muscle mutants were not structurally disorganized might be because the mutation was not strong enough to result in any structural disorganization in muscle. Alternatively, it could be due to the specific protein location that our antibodies stained while the mutation resulted in muscle disorganization in another region that our protein of interest colocalized to. For example, here we used ATN-1 to visualize the depth of dense bodies of zyx-1 mutants, yet it is reported that ZYX-1 proteins localize in the middle of dense bodies, and it might also be partially present in the basal region of the dense body where DEB-1 (vinculin) is located (60). Also, DYC-1 is known to localize at the edge of dense bodies and again DEB-1 might be a better candidate to visualize if there are any disorganizations in $d y c-1$ animals (46). It was interesting to learn that despite the fact that DYC-1 localizes close to dense bodies, we only saw mild disorganization of M-lines of $d y c-1$ mutants.

$u n c-22(s f 21)$ was one of the mutants with completely normal muscle organization, as reported earlier (51). sf21 is a missense mutation that inactivates the catalytic activity of the twitchin kinase domain, but expresses normal levels of the intact giant protein, twitchin. unc-22(sf21) mutants move faster, thus suggesting that the normal function of twitchin kinase activity is to inhibit muscle activity (51). Here, we saw that while they exert the same muscle force as WT as expected, the burrowing performance was mildly impaired. One possibility is that this impaired burrowing reflects a function of twitchin kinase in 3D but not 2D locomotion (e.g. force sensing 
of the environment). Interestingly, this muscle function and structural organization are in striking contrast to the other unc-22 allele of $e 66$, which was highly disorganized in sarcomere structure, is weak in muscle strength, and is severely impaired in burrowing. e66 is a strong loss of function allele due to a 2 bp deletion resulting in a frame-shift and premature stop codon (51). It was previously suggested that twitchin plays both regulatory and structural roles in muscle, and our results add further evidence for this contention $(13,51)$.

lev-11 encodes tropomyosin, and lev-11(x12) was another mutant with a very low muscle structural disorganization score of 1 , slight burrowing impairment, and the same strength as WT. These results could also be expected due to the regulatory function of tropomyosin during actin-myosin interaction (61). This mutation resulted in no obvious structural disorganization; however, the burrowing assay has been the only locomotory assay so far that could detect such a regulatory impairment.

\section{C. elegans as a genetic model for muscle strength}

The role of muscle strength in daily activities and exercise is indisputable. Several studies have investigated its importance in bone health (62) and its association with sarcopenia (63), heart diseases (64), and mortality (8). Age-related loss of muscle size and strength caused by a reduction in the size and number of individual muscle fibers (65) is associated with the increased frailty observed in older people (66). Given the economic impact of the aging population on healthcare systems (67), there is a greater need than ever to investigate the genetic basis of muscle strength. 
Since the body wall muscle of $C$. elegans is functionally and structurally similar to human skeletal muscle and many of the muscle genes in C. elegans are known to have human homologs, our results highlight that $C$. elegans could be used as a model organism to study the genetics of muscle strength. Moreover, $C$. elegans is an established model for muscle aging (aka sarcopenia) $(42,68-70)$. Our physiological assays of burrowing and NemaFlex can effectively assess the muscle function in mutants with genetic defects in their muscle structure. By developing muscle function and muscle disorganization scores in C. elegans, we showed a strong correlation between the two suggesting that the lower muscle function score a mutant acquires, the higher level of structural disorganization it has. This result is significant since by utilizing forward and reverse genetic screens, both the muscle physiology and muscle structure can be evaluated in C. elegans to identify the genes responsible for muscle strength. We propose that muscle function score is a valuable means to pursue such investigations.

\section{Conclusions}

Our results indicate the suitability of NemaFlex and burrowing assays to characterize muscle function of $C$. elegans. Using these testing approaches, we discuss the importance of the studied sarcomere proteins for muscle function and structure. We have shown that each of Pluronic gel burrowing assay and NemaFlex can report on different aspect of muscle physiology due to their 3D and 2D nature, stimulated and non-stimulated experimental conditions, with burrowing assay having a higher resolution in dissecting the muscle function defects.

Interestingly, when these two assays are combined, they can inform more on the muscle function and structural organization. Thus the integrated scoring methodology we have 
developed enables further evaluation of $C$. elegans as a genetic model for muscle physiology to identify conserved genes responsible for human muscle strength.

\section{Declarations}

\section{Ethics approval and consent to participate}

Not applicable.

\section{Consent for publication}

Not applicable.

\section{Availability of data and materials}

All data generated or analyzed during this study are included in this published article and its supplementary information files. The video files and images used in this study are available from the corresponding author on reasonable request.

\section{Competing interests}

S. A. V. is the co-founder of NemaLife Inc. that commercializes $C$. elegans assays. The remaining authors declare that they have no competing interests.

\section{Funding}

Some strains were provided by the CGC, which is funded by NIH Office of Research Infrastructure Programs (P40 OD010440). This work was partially supported by funding from the Cancer Prevention and Research Institute of Texas RP160806 (S.A.V), National Aeronautics 
and Space Administration NNX15AL16G (S.A.V), the Biotechnology and Biological Sciences

Research Council (BB/N015894/1 to N.J.S), and National Institute of General Medical Sciences (R01 GM118534 to G.M.B.).

\section{Authors' contributions}

L.L. and K.K. performed NemaFlex and burrowing experiments. L.L. and H.Q. conducted immunostaining and captured images. L.L. and M.N. did statistical analysis and prepared figures. L.L., H.Q., C.M.R.L, N.J.S, G.M.B, and S.A.V discussed and interpreted the results. L.L. and S.A.V. wrote the paper. All authors read, edited and approved the final manuscript. L.L., C.M.R.L, G.M.B, N.J.S and S.A.V. conceptualized the project. S.A.V. supervised the study.

\section{Acknowledgements}

We would like to thank Dr. Mathew Piasecki for useful discussions and Syed Tahsin Islam for assistance with data processing. 


\section{References}

1. Frontera WR, Ochala J. Skeletal muscle: a brief review of structure and function. Calcified tissue international. 2015;96(3):183-95.

2. Tikkanen E, Gustafsson S, Amar D, Shcherbina A, Waggott D, Ashley EA, et al. Biological insights into muscular strength: genetic findings in the UK biobank. Scientific reports. 2018;8(1):1-9.

3. Papadimitriou ID, Lucia A, Pitsiladis YP, Pushkarev VP, Dyatlov DA, Orekhov EF, et al. ACTN3 R577X and ACE I/D gene variants influence performance in elite sprinters: a multi-cohort study. BMC genomics. 2016;17(1):285.

4. Collins J, Bönnemann CG. Congenital muscular dystrophies: toward molecular therapeutic interventions. Current neurology and neuroscience reports. 2010;10(2):83-91.

5. Gao QQ, McNally EM. The dystrophin complex: structure, function, and implications for therapy. Comprehensive Physiology. 2011;5(3):1223-39.

6. Ochala J. Thin filament proteins mutations associated with skeletal myopathies: defective regulation of muscle contraction. Journal of molecular medicine. 2008;86(11):1197. 7. Seidman J, Seidman C. The genetic basis for cardiomyopathy: from mutation identification to mechanistic paradigms. Cell. 2001;104(4):557-67.

8. García-Hermoso A, Cavero-Redondo I, Ramírez-Vélez R, Ruiz JR, Ortega FB, Lee D-C, et al. Muscular strength as a predictor of all-cause mortality in an apparently healthy population: a systematic review and meta-analysis of data from approximately 2 million men and women. Archives of physical medicine and rehabilitation. 2018;99(10):2100-13. e5.

9. Zillikens MC, Demissie S, Hsu Y-H, Yerges-Armstrong LM, Chou W-C, Stolk L, et al. Large meta-analysis of genome-wide association studies identifies five loci for lean body mass. Nature communications. 2017;8(1):1-13.

10. Clark KA, McElhinny AS, Beckerle MC, Gregorio CC. Striated muscle cytoarchitecture: an intricate web of form and function. Annual review of cell and developmental biology. 2002;18(1):637-706.

11. Sleigh J, Sattelle D. C. elegans models of neuromuscular diseases expedite translational research. Translational Neuroscience. 2010;1(3):214-27.

12. Benian GM, Epstein HF. Caenorhabditis elegans Muscle. Circulation research. 2011;109(9):1082-95.

13. Gieseler K, Qadota H, Benian G. Development, structure, and maintenance of $C$. elegans body wall muscle. WormBook, ed The C elegans Research Community. 2017:1.

14. Altun Z, Hall D. Muscle system, somatic muscle. WormAtlas. 2009.

15. Qadota H, Matsunaga Y, Nguyen KC, Mattheyses A, Hall DH, Benian GM. High resolution imaging of muscle attachment structures in C. elegans. Cytoskeleton. 2017.

16. Moody JC, Qadota H, Reedy AR, Okafor CD, Shanmugan N, Matsunaga Y, et al. The RhoGEF PIX-1 directs assembly or stability of lateral attachment structures between muscle cells. Nature communications. 2020;11(1):1-15.

17. Waterston RH, Thomson JN, Brenner S. Mutants with altered muscle structure in Caenorhabditis elegans. Developmental biology. 1980;77(2):271-302. 
18. Francis GR, Waterston RH. Muscle organization in Caenorhabditis elegans: localization of proteins implicated in thin filament attachment and I-band organization. The Journal of cell biology. 1985;101(4):1532-49.

19. Francis R, Waterston RH. Muscle cell attachment in Caenorhabditis elegans. The Journal of cell biology. 1991;114(3):465-79.

20. Forbes JG, Flaherty DB, Ma K, Qadota H, Benian GM, Wang K. Extensive and modular intrinsically disordered segments in C. elegans TTN-1 and implications in filament binding, elasticity and oblique striation. Journal of molecular biology. 2010;398(5):672-89.

21. Matsunaga Y, Qadota H, Furukawa M, Choe H, Benian GM. Twitchin kinase interacts with MAPKAP kinase 2 in Caenorhabditis elegans striated muscle. Molecular biology of the cell. 2015;26(11):2096-111.

22. Zengel JM, Epstein HF. Identification of genetic elements associated with muscle structure in the nematode Caenorhabditis elegans. Cell motility. 1980;1(1):73-97.

23. Williams BD, Waterston RH. Genes critical for muscle development and function in Caenorhabditis elegans identified through lethal mutations. The Journal of cell biology. 1994;124(4):475-90.

24. Etheridge T, Rahman M, Gaffney CJ, Shaw D, Shephard F, Magudia J, et al. The integrinadhesome is required to maintain muscle structure, mitochondrial ATP production, and movement forces in Caenorhabditis elegans. The FASEB Journal. 2015;29(4):1235-46.

25. Hart AC. Behavior. WormBook, ed The C elegans Research Community. 2006.

26. Gaffney CJ, Bass JJ, Barratt TF, Szewczyk NJ. Methods to assess subcellular compartments of muscle in C. elegans. JoVE (Journal of Visualized Experiments). 2014(93):e52043.

27. Nahabedian JF, Qadota H, Stirman JN, Lu H, Benian GM. Bending amplitude-A new quantitative assay of $C$. elegans locomotion: Identification of phenotypes for mutants in genes encoding muscle focal adhesion components. Methods. 2012;56(1):95-102.

28. Hwang $\mathrm{H}$, Barnes DE, Matsunaga $\mathrm{Y}$, Benian GM, Ono S, Lu H. Muscle contraction phenotypic analysis enabled by optogenetics reveals functional relationships of sarcomere components in Caenorhabditis elegans. Scientific reports. 2016;6:19900.

29. Várkuti BH, Yang Z, Kintses B, Erdélyi P, Bárdos-Nagy I, Kovács AL, et al. A novel actin binding site of myosin required for effective muscle contraction. Nature structural \& molecular biology. 2012;19(3):299.

30. Johari S, Nock V, Alkaisi MM, Wang W. On-chip analysis of C. elegans muscular forces and locomotion patterns in microstructured environments. Lab on a Chip. 2013;13(9):1699-707.

31. Rahman M, Hewitt JE, Van-Bussel F, Edwards H, Blawzdziewicz J, Szewczyk NJ, et al. NemaFlex: a microfluidics-based technology for standardized measurement of muscular strength of $C$. elegans. Lab on a Chip. 2018;18(15):2187-201.

32. Sofela S, Sahloul S, Stubbs C, Orozaliev A, Refai FS, Esmaeel AM, et al. Phenotyping of the thrashing forces exerted by partially immobilized C. elegans using elastomeric micropillar arrays. Lab on a Chip. 2019;19(21):3685-96.

33. Beron C, Vidal-Gadea AG, Cohn J, Parikh A, Hwang G, Pierce-Shimomura JT. The burrowing behavior of the nematode Caenorhabditis elegans: a new assay for the study of neuromuscular disorders. Genes, Brain and Behavior. 2015;14(4):357-68. 
34. Lesanpezeshki L, Hewitt JE, Laranjeiro R, Antebi A, Driscoll M, Szewczyk NJ, et al. Pluronic gel-based burrowing assay for rapid assessment of neuromuscular health in $C$. elegans. Scientific Reports. 2019;9(1):15246.

35. Hewitt JE, Pollard AK, Lesanpezeshki L, Deane CS, Gaffney CJ, Etheridge T, et al. Muscle strength deficiency and mitochondrial dysfunction in a muscular dystrophy model of Caenorhabditis elegans and its functional response to drugs. Disease models \& mechanisms. 2018;11(12):dmm036137.

36. Nonet ML, Grundahl K, Meyer BJ, Rand JB. Synaptic function is impaired but not eliminated in C. elegans mutants lacking synaptotagmin. Cell. 1993;73(7):1291-305.

37. Wilson KJ, Qadota H, Benian GM. Immunofluorescent localization of proteins in Caenorhabditis elegans muscle. Myogenesis: Springer; 2012. p. 171-81.

38. Miller III DM, Ortiz I, Berliner GC, Epstein HF. Differential localization of two myosins within nematode thick filaments. Cell. 1983;34(2):477-90.

39. Benian GM, Tinley TL, Tang X, Borodovsky M. The Caenorhabditis elegans gene unc-89, required fpr muscle $M$-line assembly, encodes a giant modular protein composed of $I g$ and signal transduction domains. The Journal of Cell Biology. 1996;132(5):835-48.

40. Qadota H, Mercer KB, Miller RK, Kaibuchi K, Benian GM. Two LIM domain proteins and UNC-96 link UNC-97/pinch to myosin thick filaments in Caenorhabditis elegans muscle. Molecular biology of the cell. 2007;18(11):4317-26.

41. Ono S. The Caenorhabditis elegans unc-78 gene encodes a homologue of actininteracting protein 1 required for organized assembly of muscle actin filaments. The Journal of cell biology. 2001;152(6):1313-20.

42. Gaffney CJ, Pollard A, Barratt TF, Constantin-Teodosiu D, Greenhaff PL, Szewczyk NJ. Greater loss of mitochondrial function with ageing is associated with earlier onset of sarcopenia in C. elegans. Aging (Albany NY). 2018;10(11):3382.

43. Polet D, Lambrechts A, Ono K, Mah A, Peelman F, Vandekerckhove J, et al. Caenorhabditis elegans expresses three functional profilins in a tissue-specific manner. Cell motility and the cytoskeleton. 2006;63(1):14-28.

44. Moulder GL, Cremona GH, Duerr J, Stirman JN, Fields SD, Martin W, et al. $\alpha$-Actinin is required for the proper assembly of Z-disk/focal-adhesion-like structures and for efficient locomotion in Caenorhabditis elegans. Journal of molecular biology. 2010;403(4):516-28. 45. Han H-F, Beckerle MC. The ALP-Enigma protein ALP-1 functions in actin filament organization to promote muscle structural integrity in Caenorhabditis elegans. Molecular biology of the cell. 2009;20(9):2361-70.

46. Lecroisey C, Martin E, Mariol M-C, Granger L, Schwab Y, Labouesse M, et al. DYC-1, a protein functionally linked to dystrophin in Caenorhabditis elegans is associated with the dense body, where it interacts with the muscle LIM domain protein ZYX-1. Molecular biology of the cell. 2008;19(3):785-96.

47. Brenner S. The genetics of Caenorhabditis elegans. Genetics. 1974;77(1):71-94.

48. Qadota H, Miyauchi T, Nahabedian JF, Stirman JN, Lu H, Amano M, et al. PKN-1, a homologue of mammalian PKN, is involved in the regulation of muscle contraction and force transmission in C. elegans. Journal of molecular biology. 2011;407(2):222-31.

49. Qadota H, Moody JC, Lesanpezeshki L, Moncrief T, Kitzler D, Bhat PD, et al. A Region of UNC-89 (Obscurin) Lying between Two Protein Kinase Domains Is a Highly Elastic Spring 
Required for Proper Sarcomere Organization. Journal of Molecular Biology. 2020;432(17):4799814.

50. Mercer KB, Flaherty DB, Miller RK, Qadota $H$, Tinley TL, Moerman DG, et al. Caenorhabditis elegans UNC-98, a C2H2 Zn finger protein, is a novel partner of UNC-97/PINCH in muscle adhesion complexes. Molecular biology of the cell. 2003;14(6):2492-507.

51. Matsunaga $Y$, Hwang $H$, Franke B, Williams R, Penley $M$, Qadota $H$, et al. Twitchin kinase inhibits muscle activity. Molecular biology of the cell. 2017;28(12):1591-600.

52. Moerman DG, Plurad S, Waterston RH, Baillie DL. Mutations in the unc-54 myosin heavy chain gene of Caenorhabditis elegans that alter contractility but not muscle structure. Cell. 1982;29(3):773-81.

53. Ono S, Baillie DL, Benian GM. UNC-60B, an ADF/cofilin family protein, is required for proper assembly of actin into myofibrils in Caenorhabditis elegans body wall muscle. The Journal of cell biology. 1999;145(3):491-502.

54. Stevenson TO, Mercer KB, Cox EA, Szewczyk NJ, Conley CA, Hardin JD, et al. unc-94 encodes a tropomodulin in Caenorhabditis elegans. Journal of molecular biology. 2007;374(4):936-50.

55. Bilbao A, Patel AK, Rahman M, Vanapalli SA, Blawzdziewicz J. Roll maneuvers are essential for active reorientation of Caenorhabditis elegans in 3D media. Proceedings of the National Academy of Sciences. 2018:201706754.

56. Hewitt JE, Laranjeiro R, Norouzi M, Ellwood R, Antebi A, Szewczyk NJ, et al. Multienvironment phenotyping of $C$. elegans for robust evaluation of physical performance. bioRxiv. 2020:2020.08.17.253583.

57. Broday L, Kolotuev I, Didier C, Bhoumik A, Podbilewicz B, Ronai Ze. The LIM domain protein UNC-95 is required for the assembly of muscle attachment structures and is regulated by the RING finger protein RNF-5 in C. elegans. The Journal of cell biology. 2004;165(6):857-67. 58. Hoppe PE, Chau J, Flanagan KA, Reedy AR, Schriefer LA. Caenorhabditis elegans unc-82 encodes a serine/threonine kinase important for myosin filament organization in muscle during growth. Genetics. 2010;184(1):79-90.

59. Miller RK, Qadota H, Mercer KB, Gernert KM, Benian GM. UNC-98 and UNC-96 interact with paramyosin to promote its incorporation into thick filaments of Caenorhabditis elegans. Molecular biology of the cell. 2008;19(4):1529-39.

60. Lecroisey C, Brouilly N, Qadota H, Mariol M-C, Rochette NC, Martin E, et al. ZYX-1, the unique zyxin protein of Caenorhabditis elegans, is involved in dystrophin-dependent muscle degeneration. Molecular biology of the cell. 2013;24(8):1232-49.

61. Kagawa H, Takaya T, Ruksana R, Anokye-Danso F, Amin MZ, Terami H. C. elegans model for studying tropomyosin and troponin regulations of muscle contraction and animal behavior. Regulatory Mechanisms of Striated Muscle Contraction: Springer; 2007. p. 153-61.

62. Pang MY, Eng JJ. Muscle strength is a determinant of bone mineral content in the hemiparetic upper extremity: implications for stroke rehabilitation. Bone. 2005;37(1):103-11.

63. Manini TM, Clark BC. Dynapenia and aging: an update. Journals of Gerontology Series A: Biomedical Sciences and Medical Sciences. 2012;67(1):28-40.

64. Silventoinen K, Magnusson PK, Tynelius P, Batty GD, Rasmussen F. Association of body size and muscle strength with incidence of coronary heart disease and cerebrovascular 
diseases: a population-based cohort study of one million Swedish men. International journal of epidemiology. 2009;38(1):110-8.

65. Wilkinson DJ, Piasecki M, Atherton PJ. The age-related loss of skeletal muscle mass and function: Measurement and physiology of muscle fibre atrophy and muscle fibre loss in humans. Ageing research reviews. 2018;47:123-32.

66. Fried LP, Tangen CM, Walston J, Newman AB, Hirsch C, Gottdiener J, et al. Frailty in older adults: evidence for a phenotype. The Journals of Gerontology Series A: Biological Sciences and Medical Sciences. 2001;56(3):M146-M57.

67. Hajek A, Bock J-O, Saum K-U, Matschinger H, Brenner H, Holleczek B, et al. Frailty and healthcare costs-longitudinal results of a prospective cohort study. Age and ageing. 2018;47(2):233-41.

68. Herndon LA, Schmeissner PJ, Dudaronek JM, Brown PA, Listner KM, Sakano Y, et al. Stochastic and genetic factors influence tissue-specific decline in ageing $C$. elegans. Nature. 2002;419(6909):808.

69. Mergoud dit Lamarche A, Molin L, Pierson L, Mariol MC, Bessereau JL, Gieseler K, et al. UNC-120/SRF independently controls muscle aging and lifespan in Caenorhabditis elegans. Aging cell. 2018.

70. Christian CJ, Benian GM. Animal models of sarcopenia. Aging Cell. 2020:e13223. 